\title{
$\alpha$-Glucosidase Inhibition Assay in an Enzyme-immobilized Amino-microplate
}

\author{
Toshiro Matsui, ${ }^{\dagger}$ Mayu Shimada, Nozomi Saito, and Kiyoshi Matsumoto \\ Graduate School of Bioresource and Bioenvironmental Sciences, Kyushu University, \\ 6-10-1 Hakozaki, Higashi, Fukuoka 812-8581, Japan
}

\begin{abstract}
$\alpha$-Glucosidase (AGH) from the small intestine of rat was immobilized onto a glutaradehyde (GA) activated $\mathrm{NH}_{2}-96$ well microplate to establish a convenient and rapid AGH inhibition assay system. After AGH immobilization, remaining GA groups were blocked by $\beta$-alanine to induce a negative charge on the surface of the well. The AGH-plate showed an enzyme activity of $444 \mathrm{nU} /$ well under an assayed condition at $37^{\circ} \mathrm{C}$ for $2 \mathrm{~h}$ using $0.3 \mathrm{mM} 4$-methylumbelliferyl- $\alpha$-Dglucopyranoside as a fluorogenic substrate. Inhibitory powers of voglibose and acarbose as therapeutic AGH inhibitors were successfully evaluated to have $\mathrm{IC}_{50}$ values of 13 and $114 \mathrm{nM}$, respectively.
\end{abstract}

(Received December 22, 2008; Accepted March 11, 2009; Published April 10, 2009)

\section{Introduction}

Recent studies regarding the prevention or improvement of noninsulin-dependent diabetes mellitus (NIDDM) provide useful information that the long-term intake of $\alpha$-glucosidase (AGH, exo-type $\alpha$-D-glucoside $O$-linkage hydrolase, EC 3.2.1.20) inhibitor, acarbose, to achieve moderate blood glucose level (BGL) was effective for improving hyperglycemia in borderline NIDDM subjects. ${ }^{1}$ This indicates that achieving a high BGL close to as normal as possible through the retardation of AGH activity or inhibition of the digestive process of carbohydrates in the gut could be useful for treating diabetic patients. In this respect, we have clarified natural AGH inhibitors, including acylated anthocyanins, ${ }^{2,3}$ caffeoylquinic acids ${ }^{4}$ and theaflavins ${ }^{5}$ as an alternative medicinal food compound.

Along with screening studies of natural AGH inhibitors, we have also proposed a new immobilized AGH ( $i \mathrm{AGH}$ ) assay system to mimic the membrane-bound condition ${ }^{6}$ since AGH in mammalian intestine is anchored in the membrane by the polypeptide chain spanning the bilayer only once in the manner of $\mathrm{N}(\mathrm{in}) / \mathrm{C}$ (out) orientation ${ }^{7}$ to form a sucrase-isomaltase (SI) complex. ${ }^{8}$ The use of the $i \mathrm{AGH}$ assay system allowed the prediction of an in vivo antihyperglycemic effect of a given compound with $i \mathrm{AGH}$ inhibitory activity; compounds with less $i$ AGH inhibitory activity were no longer candidates responsible for postprandial BGL lowering, even if they showed apparent AGH inhibitory activity by a conventional free AGH assay., ${ }^{9} 10$ However, the proposed $i \mathrm{AGH}$ assay still provided great effort, because it requires each set of a reaction column containing AGH-immobilized support for each assay sample. In this study we, thus, undertook to develop a convenient $i \mathrm{AGH}$-microplate assay capable of successive evaluation of the AGH inhibitory activity in one run-in assay.

\footnotetext{
$\dagger$ To whom correspondence should be addressed.

E-mail: tmatsui@agr.kyushu-u.ac.jp
}

\section{Experimental}

\section{Materials}

Rat intestinal acetone powder was purchased from Sigma Chemical Co. (St. Louis, MO). Papain (14 U/mg-solid, from papaya latex, EC. 3.4.22.2) was a product of Nacalai Tesque (Kyoto, Japan). 4-Nitrophenyl- $\alpha$-D-glucopyranoside ( $p$ NPG) and 4-methylumbelliferyl- $\alpha$-D-glucopyranoside (MUG) were products of Sigma Chemical. Voglibose (BASEN ${ }^{\circledR}, 0.2$ $\mathrm{mg} /$ tablet) and acarbose (Glucobay ${ }^{\circledR}, 50 \mathrm{mg} /$ tablet) as synthetic AGH inhibitors were obtained from Takeda Medical Co. (Osaka, Japan) and Bayer Medical Co. (Leverkusen, Germany), respectively. A 96 well-microplate attached with a $\mathrm{NH}_{2}$ group $(0.78 \mathrm{nmol} \mathrm{cm}-2$, SUMILON-8FA, Aminoplate) or $\mathrm{COOH}$ group (0.44 nmol cm-2, SUMILON-8FC, Carboxylplate) was purchased from Sumitomo Bakelite Co. (Osaka, Japan). Other reagents were of analytical grade and used without further purification.

\section{Preparation of immobilized AGH-aminoplate}

A 96 well-aminoplate was subjected to a glutaradehyde (GA) activation treatment. The surface of each well was activated with $150 \mu \mathrm{l}$ of $2.5 \%$ GA coupling buffer $(0.2 \mathrm{M}$ phosphate buffer, $\mathrm{pH}$ 8.0) for $2 \mathrm{~h}$ at $20^{\circ} \mathrm{C}$. After being rinsed with deionized water and $50 \mathrm{mM}$ phosphate buffer ( $\mathrm{pH} 8.0), 150 \mu \mathrm{l}$ of purified AGH $(0.1 \mathrm{mg} / \mathrm{ml})$ in the coupling buffer was added to the GA-activated well. The purification of AGH from rat intestinal acetone power was done according to our previous report. ${ }^{6}$ After incubation at $20^{\circ} \mathrm{C}$ for $2 \mathrm{~h}$, the well was washed with $50 \mathrm{mM}$ phosphate buffer ( $\mathrm{pH} 8.0$ ) containing $0.5 \mathrm{M} \mathrm{NaCl}$, followed by the addition of $150 \mu \mathrm{l}$ of a $0.1 \mathrm{M}$ blocking reagent. In this experiment, four reagents ( $\beta$-alanine, 2-aminoethanol, $n$-propylamine and ethylenediamine) were used to block any remaining active aldehyde groups to introduce carboxyl $(\mathrm{COOH})$, hydroxyl $(\mathrm{OH})$, methyl $\left(\mathrm{CH}_{3}\right)$ or amino $\left(\mathrm{NH}_{2}\right)$ group onto the well, respectively. After blocking at $20^{\circ} \mathrm{C}$ for $2 \mathrm{~h}$, the support was throughly rinsed with $50 \mathrm{mM}$ phosphate buffer $(\mathrm{pH}$ 8.0) containing $0.5 \mathrm{M} \mathrm{NaCl}, 0.1 \mathrm{M}$ citrate buffer $(\mathrm{pH} 4.0)$ containing $0.5 \mathrm{M} \mathrm{NaCl}$ and subsequently $50 \mathrm{mM}$ phosphate buffer (pH 6.8). The $i \mathrm{AGH}$-aminoplate was kept at $4^{\circ} \mathrm{C}$ in the 
buffer. Immobilization of AGH $(0.1 \mathrm{mg} / \mathrm{ml})$ onto carboxylplate was performed by direct condensation with $75 \mu \mathrm{l}$ of 1-ethyl-3(3-dimethylaminopropyl)-carbodiimide (EDC) $(40 \mathrm{mg} / \mathrm{ml})$ in 20 $\mathrm{mM}$ phosphate buffer ( $\mathrm{pH} 6.0)$ at $4^{\circ} \mathrm{C}$ for $24 \mathrm{~h}$.

\section{Inhibition assay for immobilized AGH}

The immobilized AGH activity was assayed as follows. The $i$ AGH-plate was directly suspended in $200 \mu$ l of a substrate solution (50 mM phosphate buffer, $\mathrm{pH} 7.4)$. $p$ NPG $(0.7 \mathrm{mM})$ or MUG $(0.3 \mathrm{mM})$ was used as a synthetic AGH substrate for measuring the overall $i \mathrm{AGH}$ activity. After incubation at $37^{\circ} \mathrm{C}$ for $2 \mathrm{~h}$, the activity was determined by measuring the change in the fluorescent intensity on a Wallac 1420 Multilabel counter (Perkin Elmer Co., Tokyo, Japan). Excitation and emission wavelengths were set at 390 and $410 \mathrm{~nm}$ for $p$ NP detection or at 355 and $460 \mathrm{~nm}$ for MU detection, respectively. During a kinetic study, the substrate concentration was set at $0.1-1.0$ $\mathrm{mM}$ for measuring $K_{\mathrm{m}}$ and $V_{\max }$ from the Lineweaver-Burk plots. One unit of $i \mathrm{AGH}$ activity was defined as the amount of enzyme to hydrolyze $1 \mu \mathrm{mol}$ of substrate per min per well under the above-assayed conditions.

For an $i \mathrm{AGH}$ inhibition study, to the well of $i \mathrm{AGH}-m i c r o p l a t e$, $20 \mu \mathrm{l}$ of an inhibitor solution and $180 \mu \mathrm{l}$ of a substrate solution were added directly. After incubation at $37^{\circ} \mathrm{C}$ for $2 \mathrm{~h}$, the $i \mathrm{AGH}$ inhibitory activity was determined by the difference in the $i \mathrm{AGH}$ activity with or without an inhibitor. The concentration of the AGH inhibitor required to inhibit $50 \%$ of the AGH activity under the assayed condition was defined as the $\mathrm{IC}_{50}$ value.

\section{Stability test of prepared iAGH-microplate}

The stability of the prepared $i \mathrm{AGH}$-microplate activity was assessed as a function of the storage times of 1, 4, 7, 21 and 37 days after preparation. The well or $i \mathrm{AGH}$-microplate was immersed in $50 \mathrm{mM}$ phosphate buffer $\left(\mathrm{pH} \mathrm{6.8)}\right.$ ) at $4^{\circ} \mathrm{C}$ until the assay.

\section{Animal experiment}

Male 7-wk-old Sprague-Dawley rats (SPF/VAF Crj: SD, Charles River Japan, Kanagawa) were fed a laboratory diet (CE2, Clea Japan, Tokyo) and given water ad libitum. All rats were housed for 1 week at $21 \pm 1{ }^{\circ} \mathrm{C}$ and $55 \pm 5 \%$ humidity under controlled lighting from $8: 30$ to 20:30. Single oral administration of voglibose (dose; $0.07,0.34,0.76 \mathrm{mg} \mathrm{kg}^{-1}$ ) or acarbose (dose; 1.5, 2.0, $3.0 \mathrm{mg} / \mathrm{kg}$ ) was conducted in SD rats withheld for $16 \mathrm{~h}$ by gavage. After $5 \mathrm{~min}, 2 \mathrm{~g} / \mathrm{kg}$ of a substrate (maltose) solution was administered to each rat. Control rats were administered with the same substrate solution without an inhibitor. At each time point to $120 \mathrm{~min}$, about $20 \mu \mathrm{l}$ of blood sample was collected from the tail vein, being immediately subjected to a BGL measurement by a disposable glucose sensor (Glutest Pro, Sanwa Chemical Research, Co., Tokyo, Japan). The $\mathrm{ED}_{50}$ value, which is the effective dose required to achieve $50 \%$ suppression of BGL rise, was interpolatively estimated. Rat experiments in this study were carried out under the guidance of the Animal Experiment Committee of the Faculty of Agriculture and the Graduate Course of Kyushu University and the Law (No. 105, 1973) and Notification (No. 6, 1980 of the Prime Minister's Office) of the Japanese Government.

\section{Results and Discussion}

\section{Assessment of iAGH-microplate assay}

Considering that the possible immobilization amount of AGH would be extremely low towards the surface area of well

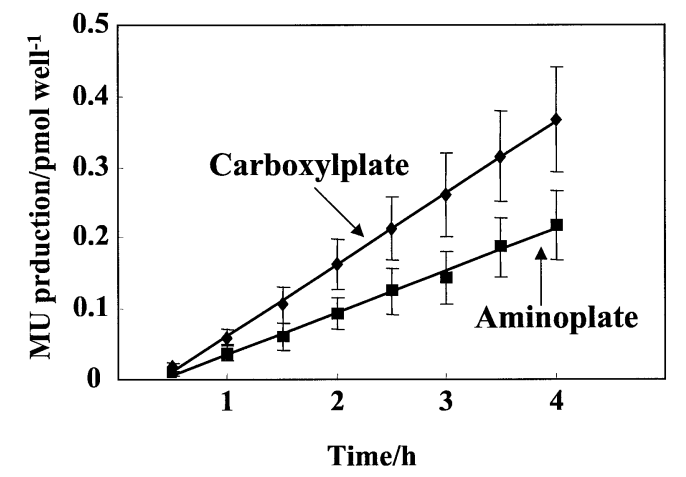

Fig. 1 Immobilized AGH activity of carboxylplate and aminoplate. 4-Methylumbelliferon (MU) from MUG as a substrate was determined for $4 \mathrm{~h}$ at $37^{\circ} \mathrm{C}$ for both $i \mathrm{AGH}$-plates. The fluorescent intensity of MU was measured at an excitation wavelength of $355 \mathrm{~nm}$ and an emission wavelength of $460 \mathrm{~nm}$. Data are expressed as mean $\pm \mathrm{SD}(n=6)$.

$\left(<1 \mathrm{~cm}^{2}\right)$, MUG with high fluorescent intensity at a level of pmol was used as a substrate throughout the study. Though data were not shown, the fluorogenic detection range of $p$ NPG, a common substrate for carbohydrases, was as narrow as $0.2-1$ $\mu \mathrm{mol} /$ assay volume.

On the basis of our previous finding 6 on preferential introduction of negative charges onto AGH-support by $\beta$-alanine to mimic the intestinal epithelium microvilli containing glycocalyx, such as sialic acid and uronic acid, ${ }^{11} i \mathrm{AGH}-$ microplate blocked by $\beta$-alanine was primarily used for optimizing the incubation time. As shown in Fig. 1, the production of 4-methylumbelliferon (MU) from MUG by $i \mathrm{AGH}$ action was linear with the incubation time to $4 \mathrm{~h}$ for both carboxylplate and aminoplate. Considering the apparent difference in the fluorescent intensity between the blank and the sample (>100000) and rapid incubation, we set at an incubation time of $2 \mathrm{~h}$ for both plates' assay in this study. The activities of $i \mathrm{AGH}$-aminoplate and $i \mathrm{AGH}$-carboxylplate were estimated to be 444 and $585 \mathrm{nU} /$ well, respectively.

\section{Catalytic property of iAGH-microplates}

The effect of the blocking reagent on the $i$ AGH activity was investigated. As summarized in Table 1, the kinetics of the prepared plates greatly differed from induced groups onto aminoplate; $\beta$-alanine and 2 -aminoethanol-induced aminoplates showed similar $K_{\mathrm{m}}$ values as carboxylplate, whereas plates blocked by ethylenediamine and $n$-propylamine provoked less affinity with MUG. The difference in the kinetics for each prepared plate may be due to the accessibility of MUG to a charged plate. Among the prepared plates, $\beta$-alanine-aminoplate did show a high catalytic efficiency $\left(v_{\max } / K_{\mathrm{m}}\right)$ of 0.08 as well as carboxylplate. This indicates that the introduction of carboxyl groups onto the surface of the plate was more favorable for the activation of immobilized AGH, being matched with an anionic environment of the intestinal epithelium microvilli by acid mucopolysaccharides. ${ }^{11}$

\section{Inhibition studies of iAGH-microplates}

Table 2 summarizes the inhibition of acarbose and voglibose against $i$ AGH-microplates with different blocking groups. Apparently, the prepared plates showed a variety of inhibitory activities with blocking groups. $\beta$-Alanine- and $n$-propylamineinduced plates showed potent $\mathrm{IC}_{50}$ values of around $20 \mathrm{nM}$ for voglibose, while no, or poor, inhibitory activity was observed in 
Table 1 Effect of blocking reagent on the kinetics of immobilized AGH onto amino- or carboxylplate ${ }^{\mathrm{a}}$

\begin{tabular}{clcccc}
\hline Plate & Blocking reagent & Functional group on the surface of plate & $K_{\mathrm{m}} / \mathrm{mM}$ & $V_{\max } / \mathrm{nmol} \mathrm{min}^{-1}$ & $V_{\max } / K_{\mathrm{m}}$ \\
\hline \multirow{2}{*}{ Amino } & $\beta$-Alanine & $-\mathrm{COOH}$ & 0.41 & 0.033 & 0.080 \\
& Ethylenediamine & $-\mathrm{NH}_{2}$ & $\mathrm{ND}^{\mathrm{b}}$ & $\mathrm{ND}$ & - \\
& $n$-Propylamine & $-\mathrm{CH}_{3}$ & 4.5 & 0.038 & 0.008 \\
& 2-Aminoethanol & $-\mathrm{OH}$ & 0.14 & 0.0026 & 0.018 \\
Carboxyl & - & $-\mathrm{COOH}$ & 0.61 & 0.070 & 0.115 \\
\hline
\end{tabular}

a. Kinetic parameters were determined from the Lineweaver-Burk plots using MUG as a substrate at a concentration range of $0.1-1.0 \mathrm{mM}$ for $2 \mathrm{~h}$ at $37^{\circ} \mathrm{C}$. b. ND, not detected.

Table 2 Effect of blocking reagent on AGH inhibitory activity of voglibose and acarbose for amino- or carboxylplate

\begin{tabular}{|c|c|c|c|c|c|}
\hline \multirow{2}{*}{ Plate } & \multirow{2}{*}{ Blocking reagent } & \multirow{2}{*}{$\begin{array}{l}\text { Functional group on } \\
\text { the surface of plate }\end{array}$} & \multicolumn{2}{|c|}{$\mathrm{IC}_{50} / \mathrm{nM}$} & \multirow{2}{*}{$\begin{array}{l}\text { Ratio of } \mathrm{IC}_{50} \text { value } \\
\text { (acarbose/voglibose) }\end{array}$} \\
\hline & & & Acarbose & Voglibose & \\
\hline \multirow[t]{4}{*}{ Amino } & $\beta$-Alanine & $-\mathrm{COOH}$ & 114 & 13 & 8.5 \\
\hline & Ethylenediamine & $-\mathrm{NH}_{2}$ & $N I^{\mathrm{a}}$ & NI & - \\
\hline & $n$-Propylamine & $-\mathrm{CH}_{3}$ & 40 & 25 & 1.6 \\
\hline & 2-Aminoethanol & $-\mathrm{OH}$ & NI & NI & - \\
\hline Carboxyl & - & $-\mathrm{COOH}$ & 110 & 270 & 0.4 \\
\hline
\end{tabular}

a. NI, no inhibition.

the other treated plates including carboxylplate. A similar inhibitory behavior in the plates was observed for acarbose. These results strongly suggested that the surface polarity of the plate induced by various blocking reagents greatly affected the inhibitory action of both inhibitors against AGH as well as the kinetic behavior (Table 1).

In the present $\mathrm{SD}$ rat experiments to evaluate in vivo antihyperglycemic potency of therapeutic AGH drugs (voglibose and acarbose), the intake of voglibose to $8 \mathrm{wk}-\mathrm{SD}$ rats showed an $\mathrm{ED}_{50}$ of $0.55 \mathrm{mg} / \mathrm{kg}$ in maltose-loaded rats, whereas a 5.6times higher dose of acarbose was required for lowering the BGL rise $\left(\mathrm{ED}_{50} ; 3.1 \mathrm{mg} / \mathrm{kg}\right.$ ) (Fig. 2). Odaka et al. ${ }^{12}$ also reported a higher antihyperglycemic potency of voglibose, rather than acarbose by a factor of 14.6 times in sucrose $(2.5 \mathrm{~g} / \mathrm{kg})$ loaded $\mathrm{SD}$ rat $\left(\mathrm{ED}_{50}\right.$ : acarbose, $5.42 \mathrm{mmol} / \mathrm{kg}$; voglibose, 0.37 $\mathrm{mmol} / \mathrm{kg}$ ). These findings indicate that the magnitude of the in vivo antihyperglycemic power of voglibose would be potentially $>5$-fold stronger than that of acarbose. From the viewpoint of the antihyperglycemic potency of both inhibitors, we re-examined the $\mathrm{IC}_{50}$ values of both inhibitors assayed by $i$ AGH-plates (Table 2). As a result, the ratio of the $\mathrm{IC}_{50}$ value of voglibose to acarbose greatly differed from induced blocking groups onto the plates; a comparable ratio of 8.5 with in vivo antihyperglycemic $\mathrm{ED}_{50}$ value was observed for the $\beta$-alanine$i$ AGH plate. A lower ratio of the $\mathrm{IC}_{50}$ value $(0.4)$ by the carboxylplate than the $\beta$-alanine-iAGH plate suggested the importance of a spacer from the $\beta$-alanine skeleton to mimic the in vivo inhibition of intestinal membrane-bound AGH. The reproducibility of the $i \mathrm{AGH}$ activity for the $\beta$-alanine-iAGH plate was $4.5 \%$ of the correlation of the coefficient $(n=8)$.

Stability of iAGH activity of the $\beta$-alanine-induced microplate

The change in the $i \mathrm{AGH}$ activity of $\beta$-alanine-aminoplate stored at $4^{\circ} \mathrm{C}$ was examined up to 37 days after preparation. As shown in Fig. 3, the prepared plate retained its AGH activity of $>60 \%$ during 21 days after preparation, while the activity disappeared at 37 days. This revealed that a reliable evaluation for AGH inhibition would be possible within 3 week-storage after preparation of the $i \mathrm{AGH}$-plate, although attempts to improve
(A)

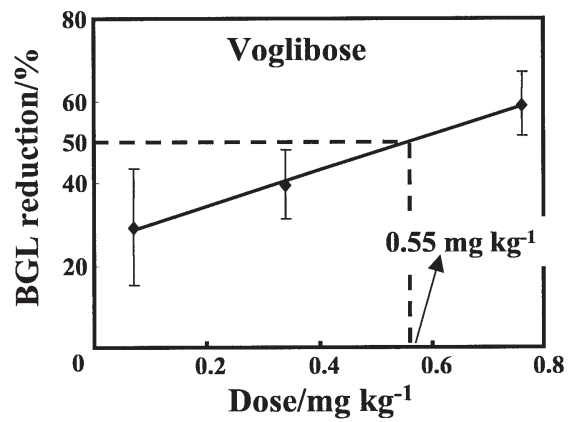

(B)

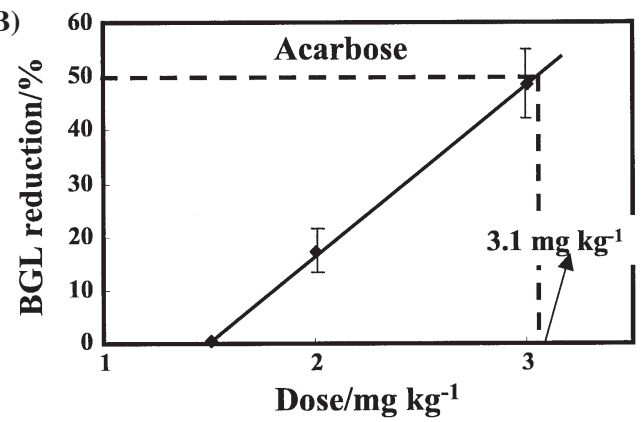

Fig. 2 Dose dependency of voglibose (A) and acarbose (B) on the reduction of glycemic responses in maltose-loaded $(2 \mathrm{~g} / \mathrm{kg}) \mathrm{SD}$ rats $\mathrm{AUC}_{0-120 \text { min }}$, which is the area under the curve of incremental blood glucose levels up to $120 \mathrm{~min}$, was used to calculate the BGL reduction ratio of each drug against control. Data are expressed as mean \pm SD $(n=4)$.

the stability of the AGH activity of the plate are needed.

Achieving BGL close to normal as possible has been considered to be one of the major goals of NIDDM therapy, in which the inhibition of intestinal AGH could be useful for improving an excess postprandial BGL rise from dietary carbohydrates. In this study, we established an $i \mathrm{AGH}-\beta$-alanineaminoplate to evaluate the inhibition of intestinal membrane- 


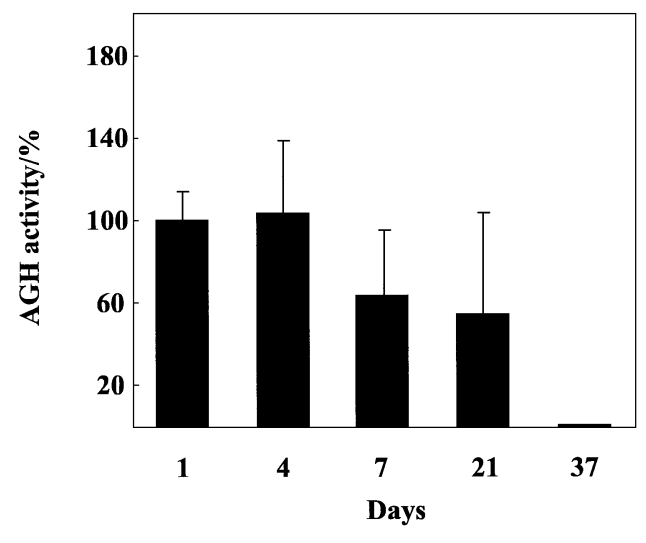

Fig. 3 Effect of the storage time on the immobilized AGH activity of $\beta$-alanine-aminoplate. The AGH activity of $i \mathrm{AGH}$-plate was individually determined on $1,4,7,21$ and 37 day-storage at $4^{\circ} \mathrm{C}$. Data are expressed as mean $\pm \mathrm{SD}(n=6)$.

bound AGH. The assay would overwhelm a conventional free AGH assay system, because of its possible estimation of an in vivo suppression effect of BGL rise by any inhibitors. The established assay was also preferable to our previous $i \mathrm{AGH}-$ Sephadex support assay, ${ }^{6}$ because of simultaneous determinations for evaluating AGH inhibition of many targeted samples in onerun-in assay.

\section{Acknowledgements}

The authors thank Mr. Masaomi Kuroki and Ms. Asami Toshima for their helpful technical support. The study was in part supported by grants from the Cooperation for Innovative
Technology and Advanced Research in Evolution Area of the Ministry of Education, Culture, Sports, Science, and Technology in Japan, and the FS Study from JST.

\section{References}

1. J. L. Chiasson, R. G. Josse, R. Gomis, M. Hanefeld, A. Karasik, and M. Laakso, Lancet, 2002, 359, 2072.

2. T. Matsui, T. Ueda, T. Oki, K. Sugita, N. Terahara, and K. Matsumoto, J. Agric. Food Chem., 2001, 49, 1952.

3. T. Matsui, S. Ebuchi, K. Fukui, K. Matsugano, N. Terahara, and K. Matsumoto, Biosci., Biotechnol., Biochem., 2004, 68,332 .

4. T. Matsui, S. Ebuchi, T. Fujise, K. J. M. Abesundara, S. Doi, H. Yamada, and K. Matsumoto, Biol. Pharm. Bull., 2004, 27, 1797.

5. T. Matsui, T. Tanaka, S. Tamura, A. Toshima, K. Tamaya, Y. Miyata, K. Tanaka, and K. Matsumoto, J. Agric. Food Chem., 2007, 55, 99.

6. T. Oki, T. Matsui, and K. Matsumoto, Biol. Pharm. Bull., 2000, 23, 1084.

7. W. Hunziker, M. Spiess, G. Semenza, and H. F. Lodish, Cell, 1986, 46, 227.

8. H. P. Hauri, H. Wacker, E. E. Rickli, B. B. Meier, A. Quaroni, and G. Semenza, J. Biol. Chem., 1982, 257, 4522.

9. T. Matsui, M. Kobayashi, S. Hayashida, and K. Matsumoto, Biosci., Biotechnol., Biochem., 2002, 66, 689.

10. J. S. Kim, C. S. Kwon, and K. H. Son, Biosci., Biotechnol., Biochem., 2000, 64, 2458.

11. Y. Muto, "Shoka to Kyushu (in Japanese)", 1988, Daiichi Publisher, Tokyo 17.

12. H. Odaka, N. Miki, H. Ikeda, and T. Matsuo, J. Jpn. Soc. Nutr. Food Sci. (in Japanese), 1992, 45, 27. 